\title{
Paravalvular leak of a mechanical mitral valve prosthesis associated with Burkholderia cepacia subacute endocarditis: a rare case successfully treated by multidisciplinary approach
}

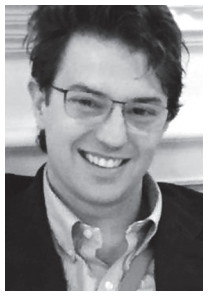

Marco Russo ${ }^{1}$, Paolo Nardi ${ }^{1}$, Guglielmo Saitto ${ }^{1}$, Pasquale Sordillo ${ }^{2}$, Dionisio F. Colella ${ }^{3}$, Massimo Andreoni $^{2}$, Antonio Pellegrino ${ }^{1}$, Giovanni Ruvolo ${ }^{1}$

${ }^{1}$ Department of Cardiac Surgery, Tor Vergata University of Rome, Rome, Italy

${ }^{2}$ Department of Infectious Disease, Tor Vergata University of Rome, Rome, Italy

${ }^{3}$ Department of Anesthesiology, Tor Vergata University of Rome, Rome, Italy

Kardiochirurgia i Torakochirurgia Polska 2017; 14 (3): 200-202

Prosthetic valve endocarditis (PVE) represents an uncommon and very serious complication after heart valve surgery. Prosthetic valve endocarditis occurs in $1 \%$ to $6 \%$ of patients with valve prostheses and affects both mechanical and biological valves [1]. Up to $34 \%$ of all cases of infective endocarditis involve prosthetic heart valves. Prosthetic valve endocarditis represents a nosographic entity independent from native valve endocarditis (NVE) because of its specific clinical features, epidemiology, and microbiological findings; its management is complex and requires a multidisciplinary approach [2]. Anatomical signs of infective endocarditis in the mitral position include valve dysfunction, paravalvular leaks, and annular abscesses. In particular, the incidence of paravalvular leaks (PVL) is estimated at $2-17 \%$ : they can be asymptomatic conditions that do not always require treatment or can cause hemolysis and heart failure [2].

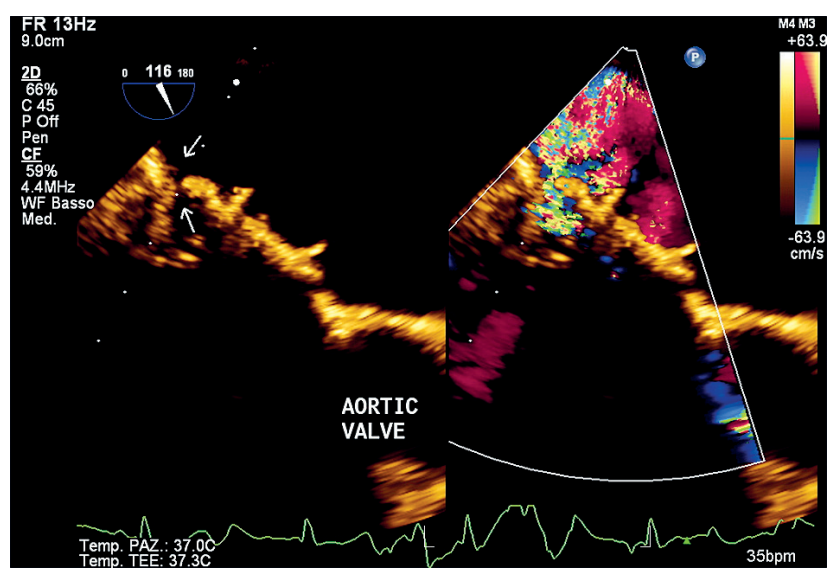

Fig. 1. TEE evidence of mitral prosthesis regurgitation. Arrows indicate the leakage
Burkholderia cepacia is a Gram-negative bacillus that represents an important nosocomial pathogen, especially in patients affected by cystic fibrosis and chronic granulomatous diseases [3]. It is rarely responsible for endocarditis in community settings, but sporadic cases have been described among intravenous heroin users and patients with prosthetic valves. According to the clinical data, most patients are treated by administration of trimethoprim-sulfamethoxazole even if the microorganism is actually characterized by multidrug resistance [4].

We present the case of a female patient who was submitted to redo cardiac surgery due to echocardiographic evidence of a paravalvular prosthetic mitral valve leak causing severe regurgitation; intraoperative evaluation revealed anatomical signs of previously undetected endocarditis, while cultures from the prosthetic valve indicated the presence of a very rare microorganism: Burkholderia cepacia.

A 75-year-old woman with history of previous mitral valve replacement with a mechanical prosthesis (St. Jude 31-mm valve in 2001) was admitted to our department with the diagnosis of prosthesis dysfunction due to a paravalvular leak and critical stenosis of the left anterior descending coronary artery. The patient was in atrial fibrillation; her medical history featured a previous stroke (2 years before).

In May 2016, the patient presented with fever and dyspnea and was admitted to the Internal Medicine Ward of one of our referral hospitals with the diagnosis of bronchopneumonia. After a thoracic computed tomography (CT) scan, an empiric antibiotic therapy with ceftriaxone and clarithromycin was administered. Due to a new onset of systolic murmur, the patient underwent transthoracic and transesophageal echocardiography (TTE and TEE), which demonstrated mitral valve prosthesis dysfunc-

Address for correspondence: Marco Russo MD, Cardiac Surgery Division, Tor Vergata University of Rome, Viale Oxford 81, 00133 Rome, Italy, phone: +39 3282528248, fax: +39 0620903536, e-mail: mar.russo1987@gmail.com

Received: 13.03.2017, accepted: 27.04.2017. 
tion due to the presence of a high trans-prosthetic gradient and a severe paravalvular leak that had not been detected during previous echocardiographic exams (Fig. 1). Coronary angiography revealed severe asymptomatic stenosis of the left anterior descending coronary artery. Blood cultures were collected (the results were negative) and antibiotic therapy was continued as prophylaxis until invasive treatment.

Due to the presence of an extensive leak not susceptible to percutaneous closure and the need for coronary revascularization, the patient was submitted to our Unit for surgical treatment. Intraoperative 3D TEE showed a very large mitral valve leak, extending counterclockwise from the antero-lateral commissure to the P2 scallop (Fig. 2). At surgical exploration, the mechanical valve appeared to be completely dehiscent with the annulus, which was completely reshaped by erosions and jagged areas. There were no vegetations or annular abscesses, but the old pledget appeared to be yellowish in color and partially destroyed (Fig. 3). The preoperative surgical strategy of surgical leak repair was promptly switched to new mitral valve replacement, which was achieved with a 25-mm SJM Regent mechanical prosthesis (St. Jude Medical, Inc., St. Paul, MN, USA). Sizing was conducted in the usual way, and a smaller prosthesis was used in accordance with the intraoperative findings. It seems that the previous valve was probably too large for the patient's native annulus in view of the large area of already existing calcification. The development of PVL could have been caused by a dual mechanism (mechanical and probably infective). Furthermore, a coronary artery bypass between the left internal thoracic artery and the left anterior descending artery was performed. The old prosthesis and pledgets were sent to the laboratory for cultures.

The postoperative course was uneventful. The patient remained afebrile with a normal blood cells count. One week after the surgery, the valve and the pledget cultures turned positive for Burkholderia cepacia; in contrast, all previous results of pre and post-operative blood cultures had been negative. Ceftriaxone and clarithromycin were replaced by cefepime ( $2 \mathrm{~g}$ every $8 \mathrm{~h}$ ). After 8 days, the patient was transferred to the Infectious Disease Unit to continue antibiotic therapy in good clinical conditions. After twenty days of antibiotic therapy, vancomycin therapy proved necessary due to the occurrence of diarrhea, which was resolved quickly. A postoperative transthoracic echocardiogram demonstrated absence of vegetation and normal function of the newly implanted mechanical valve. After one month, the patient was discharged home in good clinical condition after being advised to continue the oral antibiotic therapy (trimethoprim-sulfamethoxazole: 160/800 mg per 1 month).

After 3 months of follow-up, the patient remains free from prosthetic valve-related events, new occurrences of fever, and other symptoms of infection.

Burkholderia cepacia is a Gram-negative, aerobic, nonsporing bacillus that has been described as an important

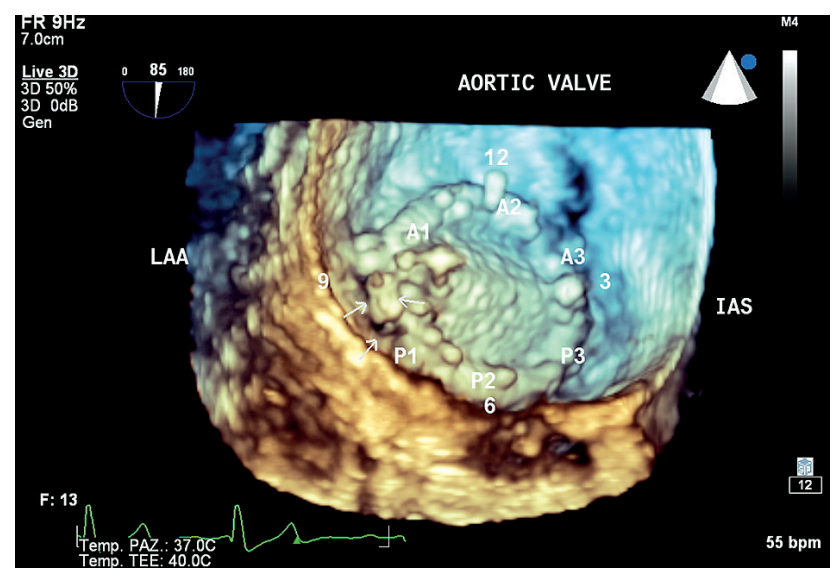

Fig. 2. 3D echo exam showing the extent of the mitral valve leakage

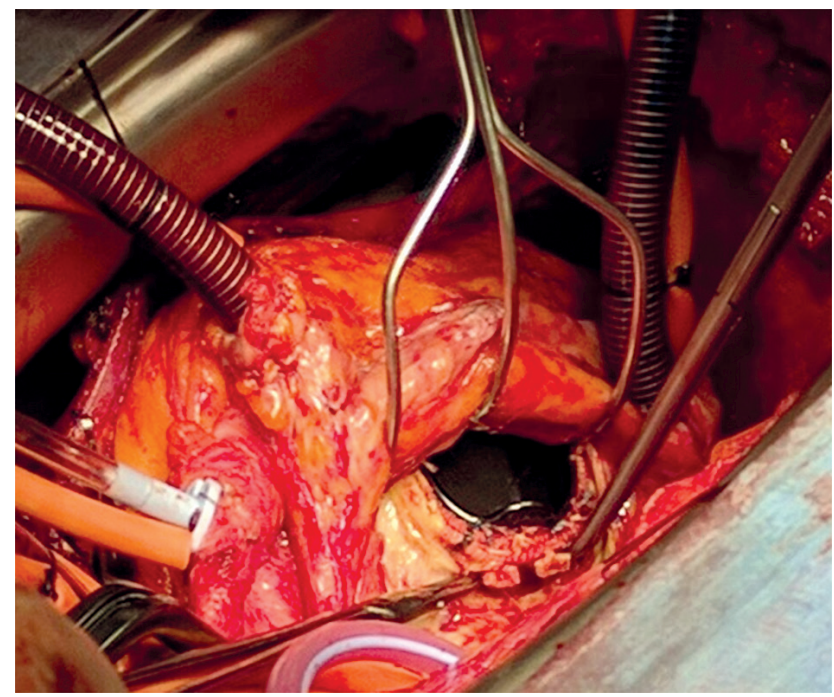

Fig. 3. Surgical findings of the endocarditis

pathogen in particular subgroups of patients (cystic fibrosis, granulomatous disease). In rare cases, it is responsible for endocarditis, especially in patients with history of heroin abuse. So far, only anecdotal cases of prosthetic valve infections caused by Burkholderia cepacia have been described [5]. Burkholderia cepacia is an intracellular parasite with poorly defined pathogenicity, which seems to determine the activation of TNF-mediated inflammation or bacteremia, causing, in rare cases, sepsis-like manifestations; infection with this pathogen is associated with a high rate of in-hospital mortality (33\%) [6]. The bacillus is an opportunistic microorganism that can invade the respiratory epithelium: in our case, the cardiac involvement may have been caused by a previous respiratory infection.

Antimicrobial resistance of Burkholderia cepacia represents a challenge. This pathogen is intrinsically resistant to aminoglycosides and polymyxins. According to a literature review of endocarditis cases caused by $B$. cepacia, published by Aggarwal et al. in 2005, most cases of infection were treated by trimethoprim-sulfometoxazole administration [5]. 
In the present case, the bacillus was resistant to even the most commonly used antibiotics, such as imipenem and ciprofloxacin. Cefepime was administered at a dose of 2 g every $8 \mathrm{~h}$.

To the best our knowledge, this is the first reported case of subacute endocarditis resulting from B. cepacia infection, causing a prosthetic heart valve leak during longterm follow-up that was successfully treated by surgical replacement and prolonged antimicrobial therapy (lasting 2 months).

Paravalvular leaks are a common complication after surgical valve replacement, with a reported incidence of 2-17\% during follow-up [7]. Among the patients in whom paravalvular leaks develop after surgery, approximately $3 \%$ require reoperations because of heart failure, hemolysis, or a combination of both. Alternative therapeutic options, such as percutaneous closure, represent a very interesting and less-invasive strategy that has recently been developed and is associated with optimal results [8].

In the era of transcatheter-based procedures, our case is a good example of how surgery can still be the preferred treatment as the surgical analysis of the prosthetic valve revealed the possible endocarditis-related etiology, while the culture of the valve allowed us to establish the diagnosis and treat the patient for the rare and unexpected subacute infection. No data in the literature define the correlation between leaks and endocarditis, and no clinical or anatomical factors, have been established to relate a leak to an infective disease in the absence of previous positive blood cultures.
According to our findings, multidisciplinary approach represents the optimal treatment strategy for patients affected by rare infective diseases involving a prosthetic heart valve.

\section{Disclosure}

Authors report no conflict of interest.

\section{References}

1. Grubitzsch H, Schaefer A, Melzer C, Wernecke KD, Gabbieri D, Konertz W. Outcome after surgery for prosthetic valve endocarditis and the impact of preoperative treatment. J Thorac Cardiovasc Surg 2014; 148: 2052-2059.

2. Tornos P, lung B, Permanyer-Miralda G, Baron G, Delahaye F, Gohlke-Bärwolf C, Butchart EG, Ravaud P, Vahanian A. Infective endocarditis in Europe: lessons from the Euro heart survey. Heart 2005; 91: 571-575.

3. Tablan OC, Chorba TL, Schidlow DV, White JW, Hardy KA, Gilligan PH, Morgan WM, Carson LA, Martone WJ, Jason JM, Jarvis WR. Pseudomonas cepacia colonization in patients with cystic fibrosis: risk factors and clinical outcome. J Pediatr 1985, 107: 382-387.

4. Livermore DM. Has the era of untreatable infections arrived? J Antimicrob Chemother 2009; 64 Suppl. 1: i29-i36.

5. Aggarwal N, Garg S, Pannu HS, Kler TS. Fatal Burkholderia cepacia early prosthetic valve endocarditis: a very rare case and a review of the literature. J Heart Valve Dis 2005; 14: 271-274.

6. Ki HK, Kim SH, Han SW, Cheong HS. A case of native valve endocarditis caused by Burkholderia cepacia without predisposing factors. BMC Infect Dis 2011; 11: 114.

7. Taramasso M, Maisano F, Denti P, Guidotti A, Sticchi A, Pozzoli A, Buzzatti N, De Bonis M, La Canna G, Alfieri O. Surgical treatment of paravalvular leak: long-term results in a single-center experience (up to 14 years). J Thorac Cardiovasc Surg 2015; 149: 1270-1275.

8. Taramasso M, Maisano F, Pozzoli A, Alfieri O, Meier B, Nietlispach F. Catheterbased treatment of paravalvular leaks. Eurointervention 2016; 12 Suppl X: X55-X60. 